\title{
Empirical behavior of a world stock index from intra-day to monthly time scales
}

\author{
W. Breymann ${ }^{1}$, D.R. Lüthi ${ }^{1, a}$, and E. Platen ${ }^{2}$ \\ 1 Zurich University of Applied Science, 8400 Winterthur, Switzerland \\ 2 University of Technology, Sydney, Australia
}

Received 19 January 2009 / Received in final form 24 August 2009

Published online 13 October 2009 - (c) EDP Sciences, Società Italiana di Fisica, Springer-Verlag 2009

\begin{abstract}
Most of the papers that study the distributional and fractal properties of financial instruments focus on stock prices or foreign exchange rates. This typically leads to mixed results concerning the distributions of log-returns and some multi-fractal properties of exchange rates, stock prices, and regional indices. This paper uses a well diversified world stock index as the central object of analysis. Such index approximates the growth optimal portfolio, which is demonstrated under the benchmark approach, it is the ideal reference unit for studying basic securities. When denominating this world index in units of a given currency, one measures the movements of the currency against the entire market. This provides a least disturbed observation of the currency dynamics. In this manner, one can expect to disentangle, e.g., the superposition of the two currencies involved in an exchange rate. This benchmark approach to the empirical analysis of financial data allows us to establish remarkable stylized facts. Most important is the observation that the repeatedly documented multi-fractal appearance of financial time series is very weak and much less pronounced than the deviation of the mono-scaling properties from Brownian-motion type scaling. The generalized Hurst exponent $H(2)$ assumes typical values between 0.55 and 0.6. Accordingly, autocorrelations of log-returns decay according to a power law, and the quadratic variation vanishes when going to vanishing observation time step size. Furthermore, one can identify the Student $t$ distribution as the log-return distribution of a well-diversified world stock index for long time horizons when a long enough data series is used for estimation. The study of dependence properties, finally, reveals that jumps at daily horizon originate primarily in the stock market while at $5 \mathrm{~min}$ horizon they originate in the foreign exchange market. The principal message of the empirical analysis is that there is evidence that a diffusion model without multi-scaling could reasonably well model the dynamics of a broadly diversified world stock index.
\end{abstract}

PACS. 89.65.Gh Economics; econophysics, financial markets, business and management - 89.75.Da Systems obeying scaling laws

\section{Introduction}

\subsection{Scaling behavior and fractal properties}

Fractal properties in financial time series, resulting in anomalous scaling, have been observed already forty years ago [1]. This picture has been confirmed and refined since 1990 [2], mainly due to the availability of intra-day data. Among the empirical stylized facts discovered are volatility clustering [3], heavy tails of return distributions with tail indices between 3 and 5 [3], anomalous scaling [3], and multi-fractal properties [4-10].

There have been a large number of studies with results that are mixed or even contradicting. One thread of research presents evidence for multifractal properties, including an analogy with turbulence [4] and the multifractal models of Mandelbrot [7-10]. The characteristic

\footnotetext{
${ }^{a}$ e-mail: luethid@gmail.com
}

empirical properties frequently discussed are the following:

- The partition sum

$$
S^{(\Delta)}(q)=\sum_{k=1}^{n(\Delta)}\left|r_{\Delta}(k \Delta)\right|^{q}
$$

of the $\log$ returns $r_{\Delta}(t) \equiv \log P(t)-\log P(t-\Delta)$ of a price series $P(t), t \in[0, T]$ display the scaling behavior [11]

$$
S^{(\Delta)}(q) \propto \Delta^{f(q)-1} .
$$

Here, $f(q) \equiv q H(q)$ is a concave function, $H(q)$ being the generalized Hurst exponent, $\Delta$ is the time horizon of the return, and $n(\Delta)=T / \Delta$. If a horizon of 1 step is chosen, the time horizon is identical with the observation time step size. Such scaling behavior reflects the essence of multifractal properties. 
- The distributions of log returns display heavy tails at short time horizons and change their form with increasing time horizon, successively approaching the normal distribution.

Such properties have been observed for both foreign exchange rates and stock indices, see e.g. the broad studies $[12-14]$ and references therein.

The models contain a kind of hierarchical structure for the volatility, which can be modeled, e.g., through a cascade structure $[5,15]$ or a multi-fractal time scale $[16,17]$. If modeling is done assuming a risk-neutral martingale measure in a diffusion setting, then the familiar machinery of risk-neutral valuation of contingent claims can be used. This requires the scaling exponent of the 2 nd moment to be one, $f(2)=1$, or, equivalently, $H(2)=0.5$.

A second thread of papers present evidence for fractional properties, which are characterized by mono-scaling behavior of the moments of absolute log returns with a linear function $f(q)$ of the form

$$
f(q)=H q
$$

with Hurst exponent $H \neq 0.5$ being independent of $q$. This implies that the distribution of log returns does not change its form with increasing time horizons. For the value of $H$, most authors find $H>1 / 2$, which signifies the existence of trends on any time scale. Mantegna and Stanley observed abnormal scaling without multi-scaling (fractional behavior) for stock market indices $[18,19]$. More recently, their findings have been confirmed by other authors for regional stock indices $[20,21]^{1}$.

In our opinion the reason for the above mixed picture is that up to now the right object has not yet been studied. Typically, foreign exchange (FX) rates, stock prices or regional stock index data are analyzed. Even though these time series seem to behave roughly similarly, there are noteworthy differences. FX rates are symmetric in the sense that there should not be any structural difference in the statistical properties between, e.g., the EUR/USD and the USD/EUR exchange rate. For stock indices, on the other hand, a crash is more likely than a sudden upwards price jump of the same size, so that one expects an asymmetric distribution. We will show further differences in this paper.

Then the question arises of what is the best object to study to obtain the best, which means most undistorted, information about a security like a currency. We argue that the best object of study is a broadly-diversified world stock index because it contains the important parts of the world market's systemic or general risk dynamics. Such an index has been constructed in reference [23]. A widelyavailable index, the MSCI-world accumulation index could also be used, see [24].

We would like to draw attention to the fact that such an object behaves differently under various aspects. In

\footnotetext{
1 Notice that both approaches assume stationary return series, while for non-stationary series with uncorrelated returns, scaling with $H(2) \neq 0.5$ would be possible [22].
}

particular, there is no transition of the form of the return distribution to a normal distribution when the time horizon is increased; this is in contrast to common believes which, at least implicitly, is present in many studies on multi-fractal properties of foreign exchange or stock market data.

The outline of this paper is as followed. Section 2 describes the data and the construction of the index. In Section 3 the results are presented. The first subsection discusses the scaling behavior together with implications on the behavior of the autocorrelation function and the quadratic variation, the second the change of the shape of the return distributions with increasing time horizon, and the third the dependence behavior on the basis of bivariate return distributions. Section 4 concludes.

\section{The data}

We study a world stock index (WSI) denominated in US dollar (USD) and eight other major currencies. For this index intra-day and daily time series are investigated.

The intra-day samples reach from 1 April 1996 to 30 June 2001. The intra-day WSI $I^{(\mathrm{WSI})}(t)$, which is described in detail in reference [23], is based on 34 country indices and the corresponding FX data. Denominated in USD it is given as

$$
I_{\mathrm{USD}}^{(\mathrm{WSI})}=\sum_{j=1}^{d} \delta^{(j)}(t) I_{\mathrm{USD}}^{j}(t)=\sum_{j=1}^{d} \delta^{(j)}(t) I_{i(j)}^{j}(t) X_{\mathrm{USD}}^{i(j)}(t) .
$$

Here, $I_{i(j)}^{j}(t)$ denotes the $j$ th local accumulation index denominated in its home currency $i=i(j)$ and held in the WSI at time $t, I_{\mathrm{USD}}^{j}(t)$ denotes the same index denominated in USD, $X_{\mathrm{USD}}^{i}(t)$ is the spot price of one unit of currency $i$ when measured in USD and $\delta^{(j)}(t)$ denotes the number of units of the $j$ th local accumulation index. The values of $\delta^{(j)}(t)$ are adjusted yearly such that they are roughly proportional to the market capitalization of the corresponding local index. The intra-day data have originally been provided by Olsen Data, who collected and cleaned them. The tick data have been regularized to 5-min data with previous-tick interpolation. Since intra-day data contain strong intra-day seasonalities of the volatility, deseasonalisation was required before further analyses.

We used a transformation onto an activity-based time scale, as described in $[25,26]$. Market activity is approximated by the weekly volatility pattern averaged over a given daylight saving period, where the definition of volatility is based on quadratic variation. Public holidays and weekends are taken into account by setting this volatility to zero. The activity-based time $\operatorname{span} \varphi\left(t_{1}\right)-$ $\varphi\left(t_{0}\right)$ is proportional to corresponding quadratic variation, and normalization is done for each daylight saving period such that $\varphi\left(t_{k+1}\right)-\varphi\left(t_{k}\right)=t_{k+1}-t_{k}$ where the $t_{k}$ are separating successive daylight saving periods. The procedure is available as S-Plus package [27]. 
The daily samples reach from 1 Jan 1973 to 10 Mar 2006. The WSI has been constructed out of 27 local indices obtained from Thomson Financial by a procedure similar to the one used for intra-day data. The number of local indices is somewhat less than for the intra-day sample because there were less indices available that date back to 1973. Weekends and worldwide public holidays have been excluded from the sample.

Notice that the introduction of EUR does not present a problem for the construction of a world index; indeed, at the date of the change to EUR, the local indices of the EUR-countries had just to be scaled by the (fixed) exchanged rates of the local currencies to EUR. We checked the procedure by verifying that no artificial jump occurred in the WSI at this date.

\section{The results}

\subsection{Scaling behavior, long-range dependence, and quadratic variation}

The scaling behavior of the partition sum has important implications on the dynamic behavior in the limit of both, very short and very long time scales. In the limit of long time scales this can be seen in the behavior of the autocorrelation function while in the short time limit it determines the behavior of the quadratic variation.

\subsubsection{Scaling behavior}

The scaling behavior of the partition sum is described by equation (2) through the function $f(q)$, which is an important characteristic of the time series. It follows from the theory of multi-fractals that this function must be concave [28]. In the simplest case, $f(q)$ is linear such that $H(q)$ reduces to the (constant) ordinary Hurst exponent $H$. For a Brownian motion $H$ takes the value $1 / 2$ while values different from $1 / 2$ are an indication for anomalous monoscaling. $H>1 / 2$ is found for time series displaying trends on any time scale, while $H<1 / 2$ is found for series displaying mean-reverting behavior (or anti-trends) on any time scale. A concave form of $f(q)$ indicates multi-scaling or multi-fractal properties.

Empirically, the scaling behavior has been determined through an estimation of the $q$ th moments of the absolute log-returns, which is obtained from the partition sums $S^{(\Delta)}(q)$ by dividing them by $n(\Delta)$. Thus, the values of $f(q)$ could be obtained through a linear regression of $\log \left(S^{\Delta} / n(\Delta)\right)$ vs. $\log \Delta$ for $q \in\{0.2, \ldots, 3\}$. Higher values of $q$ have not been included in the study because it is not certain that the corresponding moments do exist. The empirical functions $f(q)$ observed for the different cases are displayed in Figure 1. The continuous lines represent the approximation of the points by the 2nd-order expansion around the point $(0,0)$,

$$
f(q) \equiv q H(q)=c_{1} q+c_{2} q^{2}+O\left(q^{3}\right) .
$$

The values of $c_{1}$ and $c_{2}$ together with $H(1)$ and $H(2)$ are listed in Table 1.

Two remarks are in order:

1. The time horizons investigated for the high frequency sample reach from $5 \mathrm{~min}$. to one week (more than three orders of magnitudes). Time horizons for the daily sample reach from one day till 2.5 months (about two orders of magnitude). If one merges the results of the high-frequency sample and the daily sample the scaling behavior persists for four orders of magnitude. However, not for all denomination do the scaling behavior of the high frequency and the daily samples coincide, see Figure 1.

2. The quadratic term is small compared to the linear one. This means that multi-scaling is much less pronounced than the anomaly in the mono scaling. Indeed, for all but one case, the absolute value of $c_{2}$ is less than $5 \%$ of the value of the corresponding linear coefficient $c_{1}$, and about $10 \% \ldots 20 \%$ of the difference of $c_{1}-1 / 2$, see Table 1 . It is noteworthy that the scaling exponent for $q=2$ is significantly larger than 1 , namely in the range $1.1 \sim 1.2$, which means that $H(2)$ assumes values between 0.55 and 0.6. We will come back to this point below.

In the following two paragraphs the implications on the autocorrelation function and the quadratic variation are briefly discussed.

\subsubsection{Autocorrelation}

The decay of the autocorrelation function of log returns is directly related to $H(2)$ :

$$
C(\tau)=E\left[\left(r_{\Delta}(t+\tau)-\bar{r}_{\Delta}\right)\left(r_{\Delta}(t)-\bar{r}_{\Delta}\right)\right]=A \tau^{-\gamma},
$$

with exponent $\gamma=2(1-H(2))$, prefactor $A=$ $2 H(2)(2 H(2)-1)$, and $\bar{r}_{\Delta}$ denoting the expected value of the log return $r_{\Delta}(t)$. (For a simple derivation of this result we refer to the appendix.) This means that in the presence of scaling with $H(2)>0.5$ one expects long range dependence with a power law decay with decay exponents $\gamma \in(0,1)[29]$. The prefactor ensures vanishing autocorrelation for $H(2)=0.5$.

For the WSI, the empirical data show non-vanishing autocorrelations with a power law decay for both the intra-day and the daily samples. The average value of $\gamma$ is $0.63 \pm 0.10$ for the daily data and $0.83 \pm 0.29$ for the hourly data. Even though in most cases the linear regression yields better values for $R^{2}$ for the hourly samples, it is for the daily samples that the values of $\gamma$ are in somewhat better overall agreement with the values of $H(2)$. Thus, the autocorrelation function is consistent with the scaling behavior, even though there seems to be some systematic bias for hourly data. This is in line with results of Di Matteo [14] who also reported that the decay exponents of the autocorrelation are more difficult to estimate and less precise than the estimation of the function $f(q)$. 


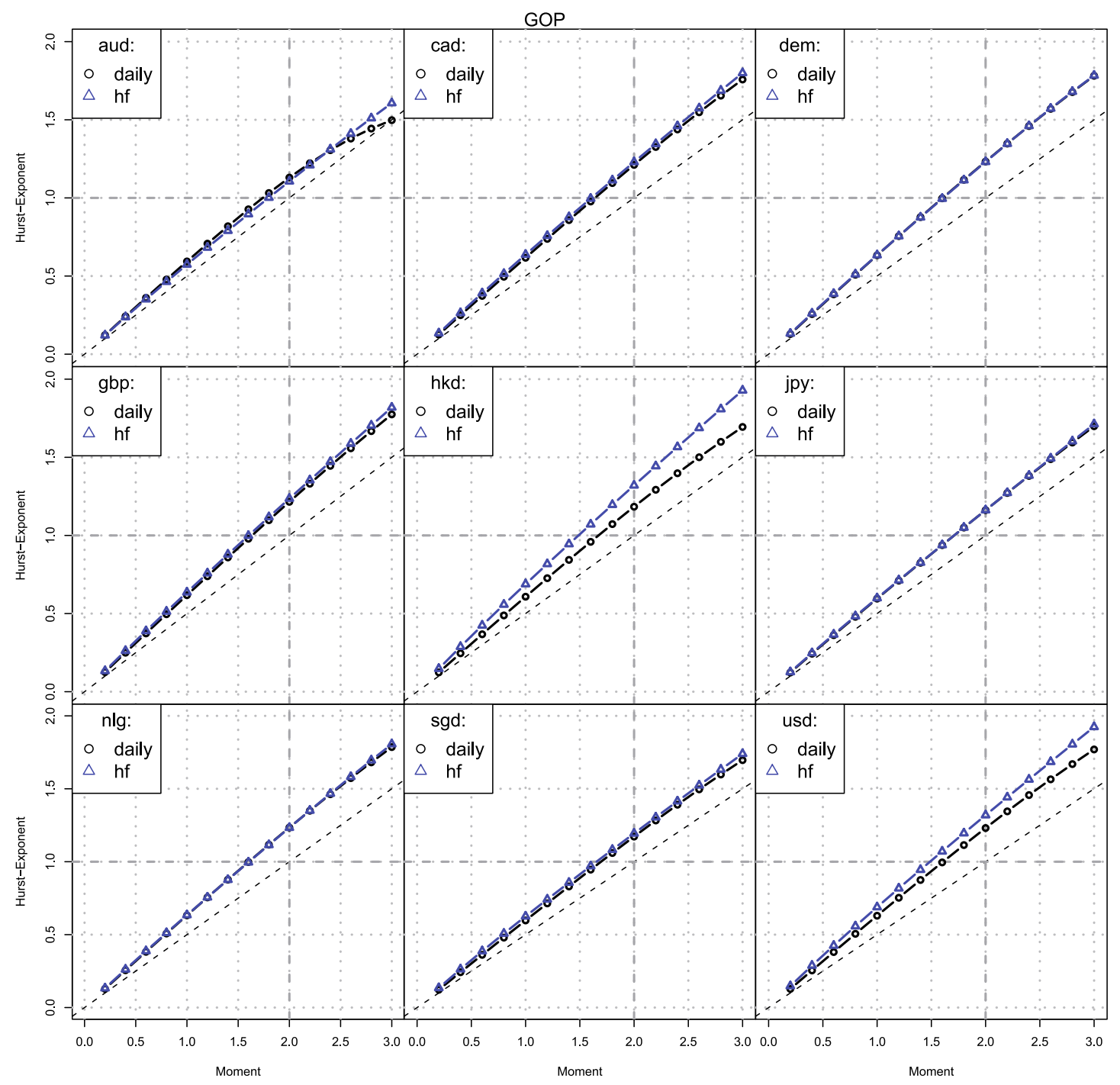

Fig. 1. Scaling exponents $f(q)$ as function of the order $q$ of the absolute moment of WSI log returns.

\subsubsection{Quadratic variation}

Quadratic variation is of central interest for stochastic processes in finance because it characterizes the fluctuations of a process. For a time-continuous process $X_{t}, t \in[0, T]$ this quantity is defined as

$$
[X]_{t}:=\lim _{\|P\| \rightarrow 0} \sum_{k=1}^{n}\left(X\left(t_{k}\right)-X\left(t_{k-1}\right)\right)^{2}
$$

where $P$ ranges over all partitions of the interval $[0, t]$, the norm $\|P\|$ denotes the length of the longest subinterval, $\|P\|=\max _{k}\left(t_{k}-t_{k-1}\right)$, and the limit is defined in the sense of convergence in probability. As we work with regular time series, $\|P\|=\Delta$ such that equation (6) can be rewritten as $[X]_{t}=\lim _{\Delta \rightarrow 0}[X]_{t}^{(\Delta)}$ with the finite size approximation

$$
[X]_{t}^{(\Delta)}=\sum_{k=1}^{n(\Delta)}\left(r_{\Delta}\left(t_{k}\right)\right)^{2}
$$

and $r_{\Delta}\left(t_{k}\right)=X\left(t_{0}+k \Delta\right)-X\left(t_{0}+(k-1) \Delta\right)$. Notice that $\Delta$ and $n=n(\Delta)$ are related by $n=t / \Delta$. Because of equation (1) this finite size approximation is precisely the partition sum, which means that the quadratic variation scales with exponent $f(2)-1$ : it vanishes for $H(2)>0.5$ and tends to infinity for $H(2)<0.5$.

The important result of our analysis is that for the WSI in all currency denominations investigated the finite-step approximation $[X]_{t}^{(\Delta)}$ of the quadratic variation tends to zero for $\Delta \rightarrow 0$.

\subsubsection{Comparison with foreign exchange rates and regional stock indices}

To summarize, the main finding is the (nearly) monoscaling of the WSI in all investigated denominations with a value of $H(2)$ in the range of $0.55 \ldots 0.6$, together with the expected implications on the behavior of the autocorrelation function and the quadratic variation. This is in 


\begin{tabular}{r|rcc|c|cccc}
\hline \multirow{2}{*}{ Curr. } & Freq. & \multirow{2}{*}{$c_{1}$} & \multicolumn{1}{c}{$c_{2}$} & $\frac{c_{2}}{c_{1}-0.5}$ & $H(1)$ & $H(2)$ & $\gamma$ & $R^{2}$ \\
\hline AUD & (daily) & 0.695 & -0.0975 & 0.500 & 0.61 & 0.560 & 0.5627 & 0.201 \\
& (hf) & 0.583 & -0.01644 & 0.198 & 0.57 & 0.555 & 0.1213 & 0.013 \\
\hline CAD & (daily) & 0.644 & -0.01807 & 0.125 & 0.62 & 0.605 & 0.6454 & 0.395 \\
& (hf) & 0.640 & -0.01462 & 0.104 & 0.64 & 0.615 & 0.8973 & 0.523 \\
\hline DEM & (daily) & 0.661 & -0.02147 & 0.133 & 0.63 & 0.615 & 0.6681 & 0.214 \\
& (hf) & 0.651 & -0.01870 & 0.124 & 0.63 & 0.615 & 1.1965 & 0.559 \\
\hline GBP & (daily) & 0.638 & -0.01467 & 0.106 & 0.62 & 0.605 & 0.8118 & 0.265 \\
& (hf) & 0.637 & -0.01124 & 0.082 & 0.63 & 0.620 & 0.8537 & 0.561 \\
\hline HKD & (daily) & 0.647 & -0.02563 & 0.174 & 0.61 & 0.590 & 0.5157 & 0.190 \\
& (hf) & 0.688 & -0.01695 & 0.090 & 0.69 & 0.660 & 0.9811 & 0.718 \\
\hline JPY & (daily) & 0.608 & -0.01392 & 0.129 & 0.60 & 0.580 & 0.6108 & 0.386 \\
& (hf) & 0.596 & -0.00991 & 0.103 & 0.60 & 0.580 & 0.8143 & 0.600 \\
\hline NLG & (daily) & 0.660 & -0.02066 & 0.129 & 0.63 & 0.615 & 0.5805 & 0.333 \\
& (hf) & 0.640 & -0.01323 & 0.095 & 0.63 & 0.615 & 0.7980 & 0.658 \\
\hline SGD & (daily) & 0.625 & -0.01848 & 0.148 & 0.60 & 0.585 & 0.5041 & 0.291 \\
& (hf) & 0.623 & -0.01647 & 0.134 & 0.62 & 0.600 & 0.8847 & 0.526 \\
\hline USD & (daily) & 0.666 & -0.02366 & 0.143 & 0.63 & 0.615 & 0.7353 & 0.307 \\
& (hf) & 0.689 & -0.01775 & 0.094 & 0.69 & 0.660 & 0.9460 & 0.813 \\
\hline
\end{tabular}

Table 1. Behavior of the scaling exponents for log returns of the WSI denominated in different currencies. Both $H(1)$ and $H(2)$ are significantly larger than 0.5. The deviation of the linear part from Brownian-motion scaling $(H=0.5)$ is about ten times larger than the quadratic term. This means that anomalous mono scaling (the mono-fractal part) is the predominant part, and the multi-fractal part can be considered as a higher-order correction. Notice that the scaling exponent of the second moment is significantly larger than 1.

contrast to the behavior of both, regional (country-based) stock indices and foreign exchange $(\mathrm{FX})$ rates. Indeed, multi-scaling is typically reported for both, FX rates and stock indices, see e.g. [12-14]. The major difference between stock indices and FX rates is that the scaling behavior of stock indices display a remarkable difference between developed and emerging markets, $H(2)<0.5$ for the former and $H(2)>0.5$ for the latter. For FX rates there is a overall tendency of the values of $H(2)$ to cluster closer around the value 0.5 , however with notable exceptions as the USD/HKD rates, which can be explained by the fact that for a number of years the Hong Kong dollar has been pegged to the $\mathrm{USD}^{2}$.

\subsection{Shapes of the return distributions}

For characterizing the return distributions $f_{R}(r)$ the empirically observed log-returns $r$ have been fitted with generalized hyperbolic (GH) distributions by means of maximum-likelihood estimation for all data sets. GH distributions, which have been introduced by BarndorffNielsen [30,31], provide an excellent class of distributions to faithfully parameterize the great majority of return distributions encountered in financial markets [32-35]. Their

\footnotetext{
${ }^{2}$ Indeed, if a currency is pegged to another, for the corresponding FX rate $H(q)$ will decrease to values smaller than 0.5 . The reason is that pegging requires corresponding interactions of the central bank, which generates a mean-reversing effect of the corresponding rate. A similar effect has been observed in the 90th for the European currencies tied together in the EMS, see reference [3].
}

role in financial modeling is discussed e.g. in reference [36]. They belong to the class of mean-variance mixture distributions whose density is given as

$f_{R}(r)=\int_{0}^{\infty} \frac{e^{\frac{r-\mu}{\sigma^{2}} \gamma}}{\sqrt{2 \pi} \sigma} \exp \left\{-\frac{(r-\mu)^{2}}{2 \sigma^{2}} \frac{1}{w}-\frac{1}{2} \frac{\gamma^{2}}{\sigma^{2}} w\right\} h(w) d w$.

Here $\mu$ and $\sigma$ are location and scale parameters, $\gamma$ denotes the skewness parameter, and $w$ describes the meanvariance mixture. For the class of generalized hyperbolic distributions $h$ is the generalized inverse Gaussian density

$$
h(w)=\left(\frac{\psi}{\chi}\right)^{\lambda / 2} \frac{w^{\lambda-1}}{2 K_{\lambda}(\sqrt{\chi \psi})} \exp \left\{-\frac{1}{2}\left(\frac{\chi}{w}+\psi w\right)\right\}
$$

where $K_{\lambda}$ denotes the modified Bessel function of the $3 \mathrm{rd}$ kind. The density $h$ depends on the three parameters $\chi$, $\psi$, and $\lambda$. The closed form density of the GH distribution in this parametrization can be found in [35]. In fact, the problem is over-parametrized, so that an additional condition must be imposed. Here, we choose $\int w h(w) d w=1$, which makes it possible to substitute $\chi$ and $\psi$ by a single parameter $\bar{\alpha}$ through the relationship

$$
\chi=\bar{\alpha} \frac{K_{\lambda}(\bar{\alpha})}{K_{\lambda+1}(\bar{\alpha})}, \quad \psi=\frac{\bar{\alpha}^{2}}{\chi}=\bar{\alpha} \frac{K_{\lambda+1}(\bar{\alpha})}{K_{\lambda}(\bar{\alpha})},
$$

so that there are only two independent shape parameters. For the symmetric case this parametrization has already been used in reference [37].

The GH class comprises distributions of the following types: variance gamma (also called generalized 

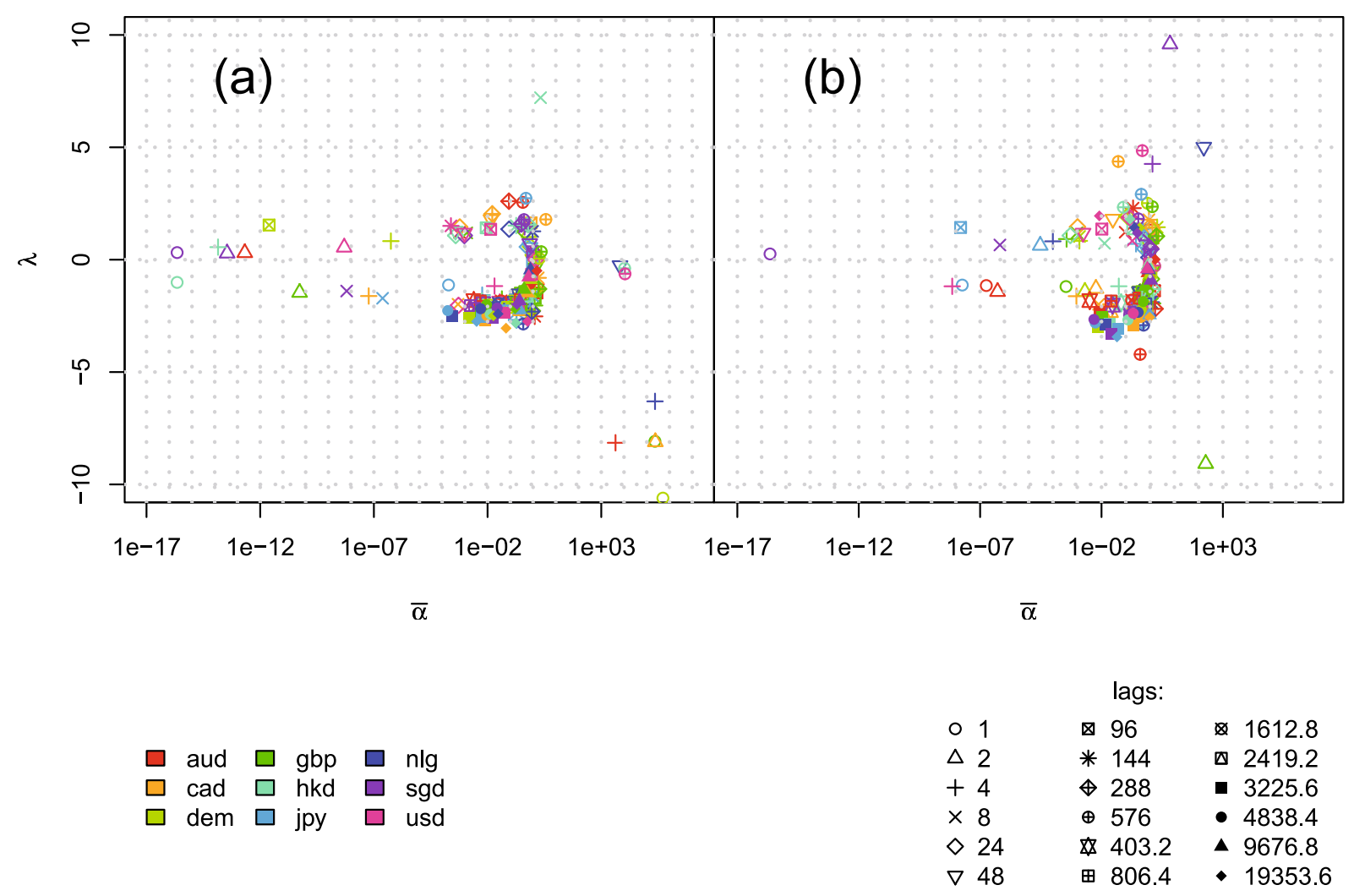

Fig. 2. $\bar{\alpha}-\lambda$ diagram for fit of empirical log returns of the WSI expressed in different currencies to SGH-distributions for time horizons from 5 min to 2.5 months.

Laplace distribution [38]), hyperbolic [39], normal inverse Gaussian [40], and Student-t. They are consistent with different types of processes used for modeling the price dynamics, e.g. Lévy-processes [41-43] and the Minimal Market Model [44]. GH distributions have semi-heavy tails with the exception of the Student- $t$ limiting case, which has heavy tails.

The values of the shape parameters $\bar{\alpha}$ and $\lambda$ are shown in Figure 2 fitted to symmetric (left) and skewed (right) GH distributions. In this plot, the deviation from the normal distribution increases with decreasing distance from the center of the coordinate system i.e., the normal distribution is obtained in the $|\lambda| \rightarrow \infty$ or/and $\bar{\alpha} \rightarrow \infty$ limit. The upper $\bar{\alpha}=0$ half line represents the variance gamma (VG) distribution and the lower half line represents the Student- $t$ distribution with $\nu=-2 \lambda$ degrees of freedom. Notice that the $\bar{\alpha}$-axis is plotted on a logarithmic scale, which enlarges very short distances from the $\bar{\alpha}=0$ line. The WSI currency denominations are encoded by the colors of the symbols and the time horizons by their shapes, the unfilled forms being used for small time horizons and the filled ones for long horizons.

The overall shape of the sets of points is similar for the fit with symmetric distributions (left) and skewed distributions (right). This is an indication of the fact that skewness plays little role. Indeed, a closer look on the $\gamma$-values revealed that only very small negative skewness is found with $\gamma$-values contained in the interval $[-0.01,0]$ (and clustering near 0 ) for the majority of cases and $\gamma<-0.03$ only for some exceptional cases. Correspondingly, skewness is not discussed in further detail.

There is a significant concentration of points for $\bar{\alpha}$ practically zero or very small and $\lambda$ either between 1 and 2 (VG) for the very short horizons of the order of an hour or less and between -2 and -3 (Student- $t$ distribution between 4 and 6 degrees of freedom) for the very long time horizons of up to several months; on average there is a smooth transition from one limiting case to the other passing on a curved line.

The transition towards the $t$-distribution for long time horizons is corroborated by Figure 3, which displays goodness of fit results. Indeed, the difference of the Akaike information criterion (AIC) of the GH or $t$ distributions for both, the symmetric and asymmetric case, and the normal distribution is always negative, indicating that these distributions provide a better fit. This is true even for time horizons as long as 2.5 months. Only at the largest time horizon of the intra-day sample, the distribution is very close to a normal distribution, which is probably due to the fact that the intra-day sample covers a time span of only about five years.

More important, for long time horizon, the $t$ distribution turns out to provide at least as good results as the more general GH distribution, and the differences between the symmetric and asymmetric case, if any, are very small. 


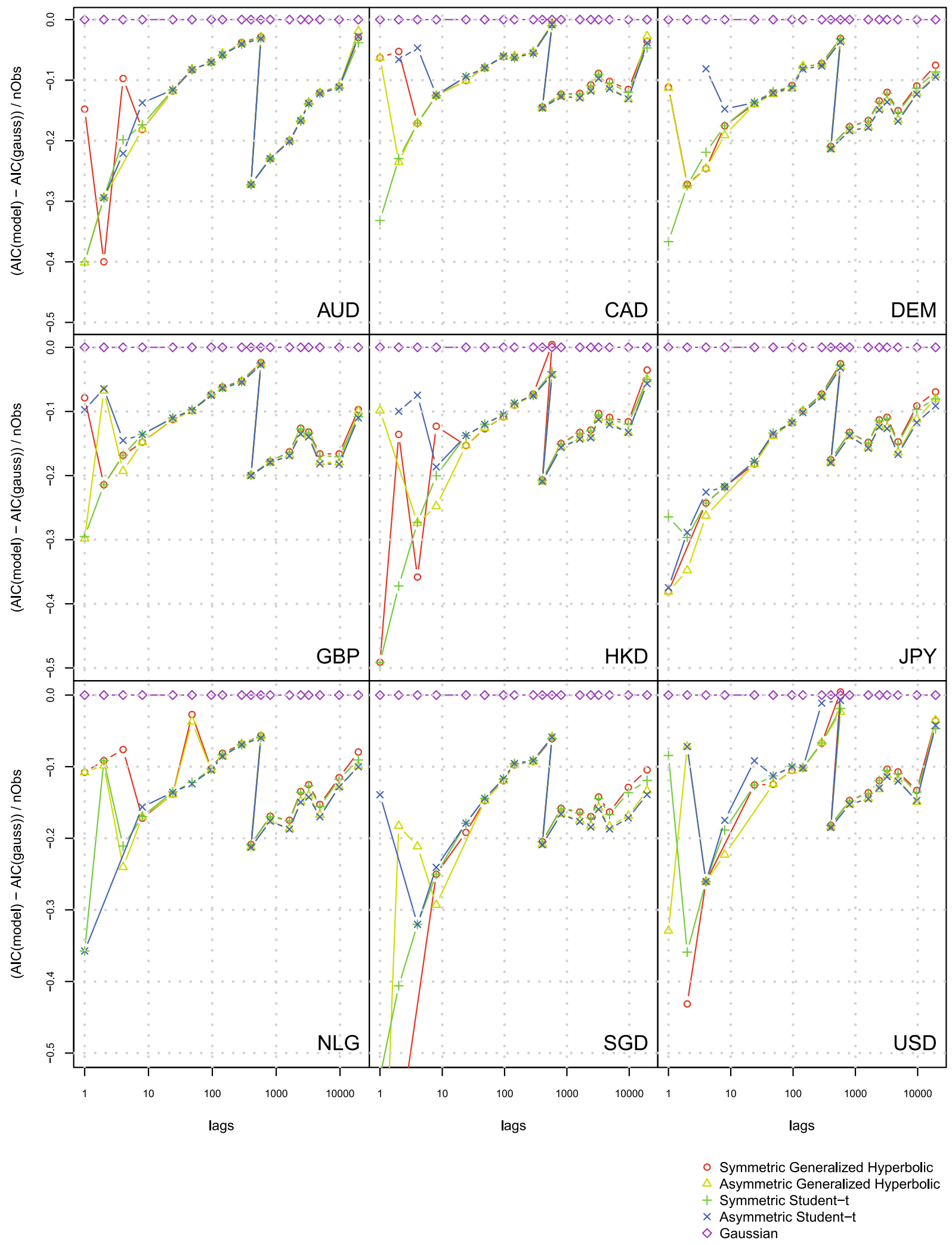

Fig. 3. Goodness of fit relative to the normal distribution for the of WSI log returns computed for different time horizons and denominated in different currencies. The data are fitted to symmetric and asymmetric generalized hyperbolic and student- $t$ distributions. The difference $\mathrm{AIC}_{\text {Model }}-\mathrm{AIC}_{\text {Gauss }}$ is scaled by the number of observations to make values obtained for different time horizons comparable (cf. [26]). More negative values correspond to better fits. 
Our results are in line with results reported for intraday [23] and daily [37] data. Let us recall that the Student- $t$ distribution is the only limiting case displaying heavy tails. The log returns remain $t$-distributed with degrees of freedom between 4 and 6 when the time horizon is increased up to several months. This means that for a broadly based world stock index there is no transition from heavy-tailed return distributions to normal distributions when enlarging the time horizon, contrary to what has been suggested by other findings on regional indices or FX data.

In the following section, we give some hints how the aggregate behavior of different regional stock indices and different FX rates could generate such a behavior.

\subsection{Dependence structure}

According to equation (4) the dynamics of the WSI expressed in a certain currency is the aggregated dynamics of many country indices and the corresponding FX rates of the local currencies with respect to the currency used as denomination. To understand the influence of the different constituents it would be helpful if one could separate them. This can be done to a certain extend by comparing the WSI expressed in different denominations. A comprehensive analysis along this line is quite extensive and well beyond the scope of this paper. Here we will take only some explorative steps into this direction, which already yields quite interesting insight into the effects which may cause the stylized facts exposed in the previous sections.

In the following we concentrate on understanding the role of the FX dynamics in this picture. In fact, the contribution of a single FX rate can be separated in the following way. Let $I_{\mathrm{k}}^{(\mathrm{WSI})}$ be the WSI denominated in an arbitrary currency $k$. Its construction is given by replacing USD by the arbitrary currency $k$ in equation (4). Then the transformation to the denomination in a third currency $l$ is given as

$$
\begin{aligned}
I_{l}^{(\mathrm{WSI})}(t) & =\sum_{j=1}^{d} \delta^{(j)}(t) I_{i(j)}^{j}(t) X_{l}^{i(j)}(t) \\
& =\left(\sum_{j=1}^{d} \delta^{(j)}(t) I_{i(j)}^{j}(t) X_{k}^{i(j)}\right) X_{l}^{k}(t) \\
& =X_{l}^{k}(t) I_{k}^{(\mathrm{WSI})}(t) .
\end{aligned}
$$

Due to this relationship the log return of the WSI denominated in a currency $l$ is simply given by the sum of the WSI denominated in currency $k$ and the corresponding FX log return,

$$
r_{\Delta}^{I_{l}}(t)=r_{\Delta}^{I_{k}}(t)+r_{\Delta}^{k / l}(t)
$$

To get an idea about the influence of a single currency in the WSI we undertook pairwise comparison of the WSI returns denominated in different currencies, see scatter plot 4 and 5 . Note, that the color encoding of these plots represents the bivariate density on a logarithmic scale. For details the reader is referred to the captions.

Figure 4 displays the densities for daily WSI returns, where for all plots the scales of the axes are the same. The following facts are striking:

(i) in all denominations the volatility is approximately the same;

(ii) the principal axes are all aligned along the diagonal;

(iii) the densities seem to be composed of an elliptical contour, with some extrem events (jumps) principally located on the diagonal; and

(iv) there are more downward jumps than upward jumps.

The first two facts imply that for the dynamics of the WSI any single FX rate has a minor effect relative to the aggregate behavior of the country-based indices and the other FX-rates, which remain unchanged by transformation (11). This fact is hardly surprising. (If the separated FX rate had no influence at all, one would have complete dependency of the returns expressed in different denominations such that the bivariate density would degenerate to a univariate one. This can nicely be seen in the plot of the denominations DEM vs. NLG, the latter having practically been pegged to DEM.) The extreme events originating in the disentangled FX-rate can be detected as individual points not located on the diagonal. Particularly visible are some such points for the WSI denominated in AUD and in HKD. Since most of the extreme events are located on or near the diagonals they do not originate in the FX rates considered in this figure (i.e., any combination of the currencies AUD, CAD, DEM, GBP, HKD, JPY, NLG, SGD, USD). If we suppose that the results would be similar for the FX rates not studied here, the extreme events must originate in the different country-based stock markets ${ }^{3}$.

Finally we note that fact (iv) may impact the scaling properties if this feature persists on different time scales.

At the $5 \mathrm{~min}$. observation time step size displayed in Figure 5 things look quite differently. Here, again, the WSI return densities are all plotted with the same scales of the axes. The following facts are striking:

(v) Again, the volatility is approximately the same for all denominations.

(vi) The principal axes are aligned along the $x$ and/or $y$ axes.

(vii) The extreme events are aligned along the $x$ and/or $y$ axes.

The loss of correlations or (more generally) dependency in the deep intra-day regime is a well-known stylized fact.

\footnotetext{
3 In fact, there would still be the possibility that all the exchange rates with respect to some special currency are driven by a single risk driver. In practice this should be closely related to the USD. In this case the scatter plots where one of the denominations is the USD should look different from the other plots. It can easily be checked in Figure 4 that this is not the case. In addition, we checked on the scatter plot of FX rates that the (very improbable) hypothesis of a single main risk driver is indeed excluded.
} 


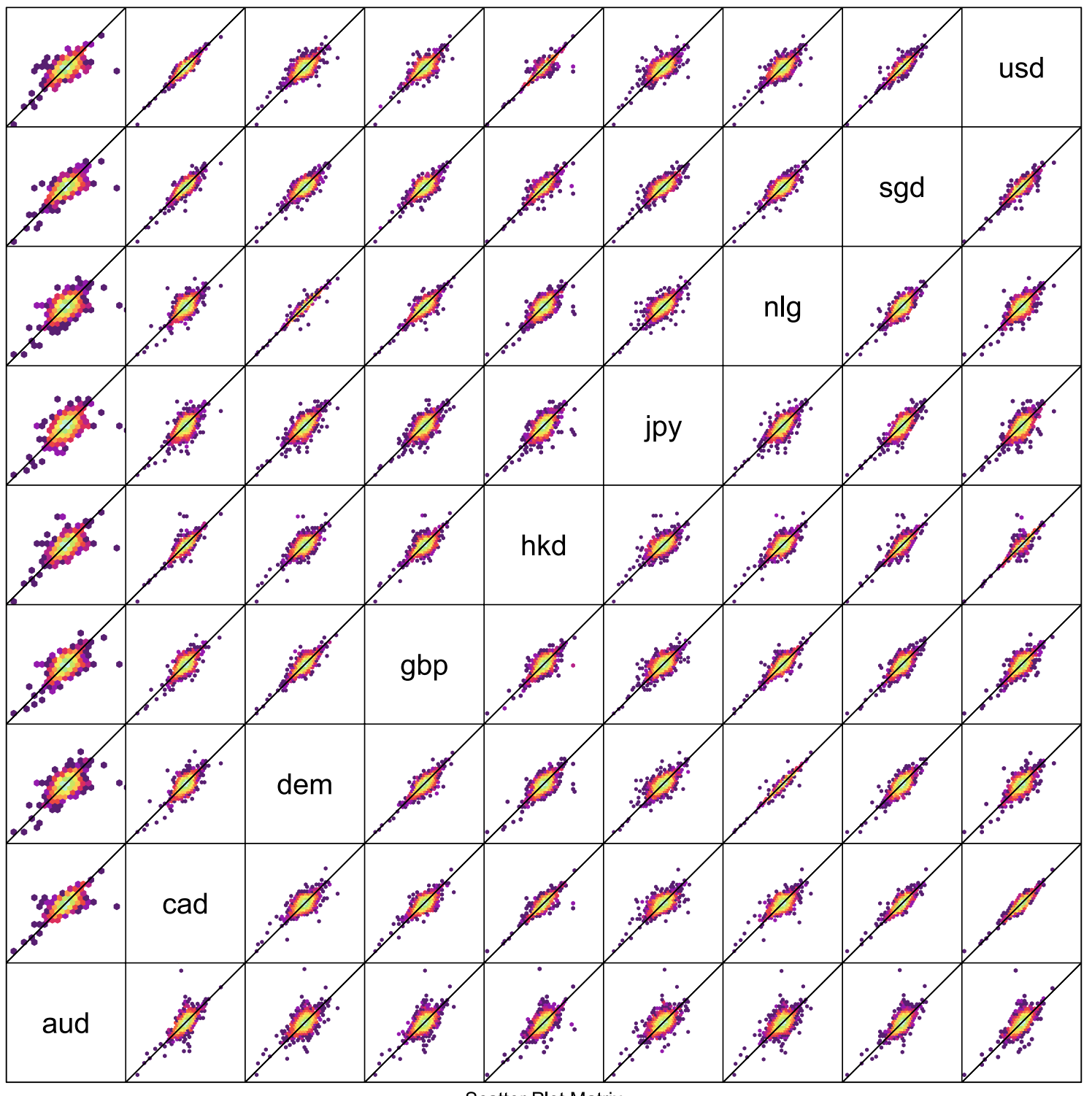

Fig. 4. Color-encoded density plots of bivariate daily WSI log returns. The colors are (ordered by increasing values) purplered-orange-yellow-green. All axes have the same scale, reaching from -0.11 to 0.11 . The density is represented on a log scale. Notice that the bivariate densities are all aligned along the diagonals and have comparable shapes. Also outliers are mainly aligned along the diagonals.

However, in view of equation (12) it follows from (vi) and (vii) that now the main contribution must stem from the FX rates. Indeed, otherwise the dependency should persist independent of the time horizon! This is true for both, the linear correlation as well as for the jumps. It means in particular that at the 5 min. time horizon the jumps originate in the FX market. It is only at larger time horizon that extreme events originate in the stock market.

The fact that at the daily time horizon the FX dynamics plays a minor role in the WSI dynamics while at the $5 \mathrm{~min}$. time horizon it seems to dominate this dynamics seems puzzling at first sight. However, the difference in the scaling exponents explains this fact. Indeed, with a value of $H=0.6$, the ratio between the daily and the 5 min. return for the WSI is about 30 while with $H=0.5$ for the FX return this factor is typically around 17 , i.e., only half that value. This means that with decreasing time horizon FX returns decrease slower than WSI returns and consequently gain in relative importance.

\section{Discussion and conclusion}

The following facts have been observed for the log returns of a WSI denominated in different currencies.

- Mono-scaling properties with $H=0.55 \sim 0.6$, together with the related power law decay of the autocorrelation function (acf) and scaling of the quadratic variation in the $\Delta \rightarrow 0$ limit. 


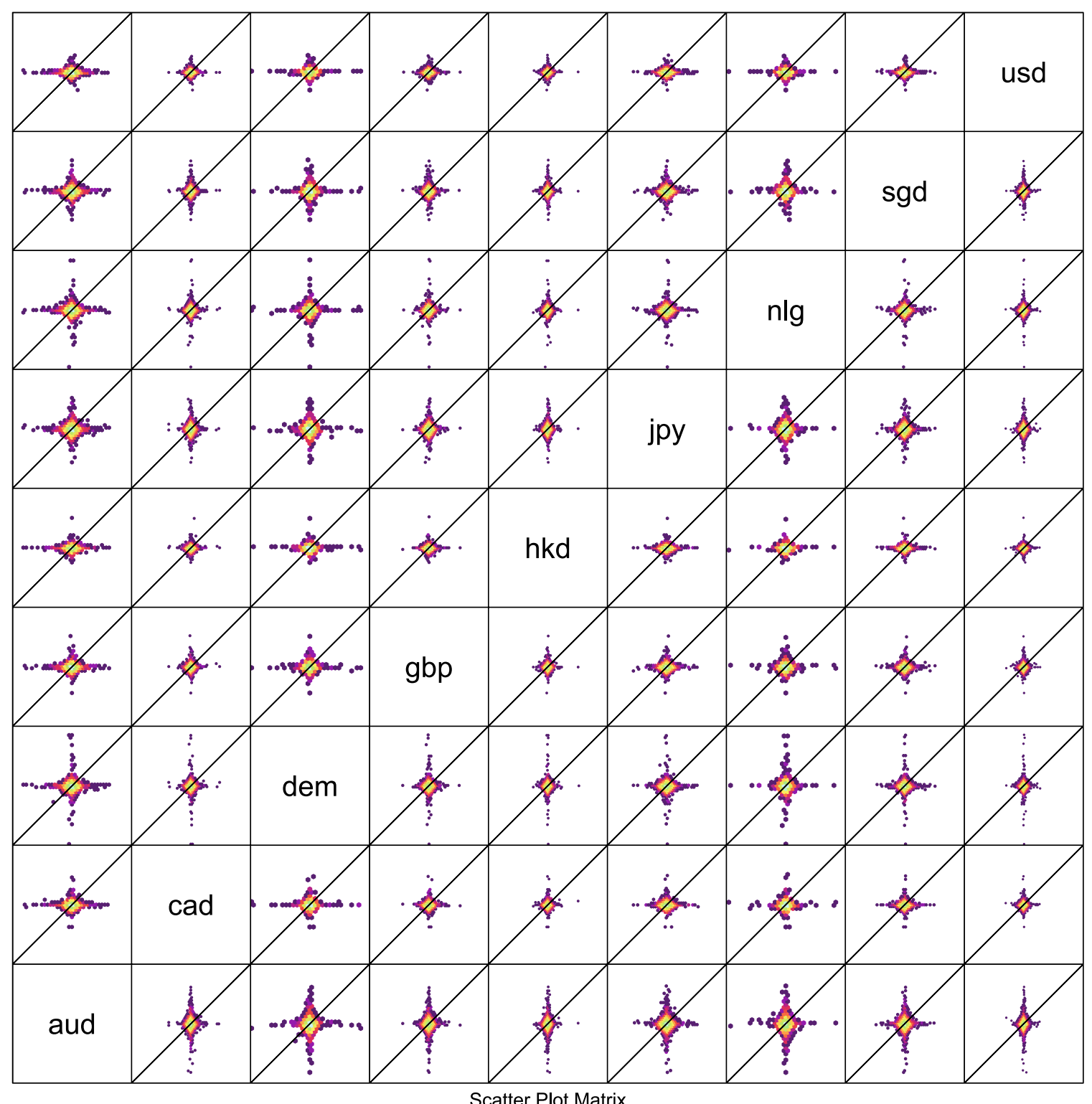

Fig. 5. Same as Figure 4 for $5 \mathrm{~min}$ WSI $\log$ returns. The scale of the axes reaches from -0.013 to 0.013 . Again, the shapes of all the densities are similar but now the principal axes are the $x$ - and $y$-axis, and outliers are aligned along theses axes.

- Variance-gamma distribution for small time horizons, which successively transform into a Student- $t$ distribution for larger observation time step sizes.

- The dynamics at daily horizon is dominated by the stock markets. In particular, jumps mainly originate in the stock markets and only rarely in the FX markets.

- At the 5 min. horizon, the FX dynamics is much more important (perhaps even dominant) for the characterization of the WSI dynamics. In particular, jumps observed at that time horizon originate in the FX market.

From these observations it follows that the interesting scaling properties of the WSI, together with the absence of a transition of the return distribution towards normaldistribution for large observation time steps, is likely to be caused by a combination of events. On one hand, the high-frequency small FX jumps drive the WSI dynam- ics at very short intra-day time scales. At larger (daily and longer) time horizons, jumps occur in the stock markets. These jumps are larger and less frequent. There may even be a hierarchy of jumps which is required to generate the almost mono-scaling behaviour. The existence of such a hierarchy is suggested by the fact that the stock market is driven by information on a weekly, monthly or even quarterly scale with groups of market participants acting on a similar time scale. Such a process would be able to generate the observed scaling behaviour and thus also the observed behaviour of the quadratic variation, even though it could still be described in the framework of jump-diffusion processes.

At the current state, however, it is not certain that the empirical behaviour of WSI returns presented here is entirely caused by jumps. Delay effects, which may be caused by the time needed for institutional or other groups 
of investors to react to the information and rebalance their portfolio, may likewise play a role. To clarify these points, further research is needed.

As to the theoretical description, it is too early to say which kind of approach will eventually prevail. The convincing integration of the above-mentioned empirical findings on financial market data into a unifying theoretical framework for the entire financial market is still missing. Mathematical theories which aim at a generalization of standard processes towards Lévy processes and fractional Brownian motion, are typically formulated for a single asset, and are still in their infancy as far as modeling the entire market dynamics is concerned [43]. We would like to mention, however, that at present the most promising theory to described such an object appears to be the Benchmark approach [44], which is a continuous time diffusion framework with stochastic diffusion and drift coefficients. Optimization of log growth leads to the growth optimal portfolio (GOP), which has been shown to be approximated by any broadly diversified capital market index in the limit of infinitely many instruments. Under some additional technical assumptions the log returns are expected to be $t-4$ distributed, independent of time scale. However, it has to be seen how the theory can be extended in a way to account for the observed mono-fractal scaling behavior.

What we have shown in the current paper is that a broadly diversified index has a much better signal to noise ratio than, e.g., stock prices or exchange rates. The diversification effect provides cleaner statistical properties for an index, which has shown to be very useful to disentangle otherwise bewildering facts.

We thank Renata Rendek for her inspiring remarks and Juri Hinz for some clarifying discussions. We are also indebted to René Locher and Simon Otziger for valuable comments and help concerning the presentation of the data. This research has been financially supported by the COST P10 action and Swiss SER grant no. C05.0039.

\section{Appendix A: Return autocorrelation functions for multi-fractal price series}

Let $\Delta$ denote the elementary time step, e.g. the sampling period. The return with lag $k$ is defined as

$$
r_{k}(t)=x(t)-x(t-k \Delta) .
$$

From the aggregation property of returns, $r_{k}(t)=$ $\sum_{i=0}^{k-1} r_{1}(t-i \Delta)$ and the "stationarity" of $r_{i}(\cdot)$ it immediately follows that

$$
\begin{aligned}
E\left(r_{k+1}(t)^{2}\right)= & E\left(r_{k}(t)^{2}\right)+E\left(r_{1}(t)^{2}\right) \\
& +2 \sum_{i=0}^{k-1} E\left(r_{1}(t-k \Delta) r_{1}(t-i \Delta)\right) .
\end{aligned}
$$

The acf for a process with zero mean is defined as

$$
C(i)=\frac{E\left(r_{1}(t) r_{1}(t-i)\right)}{E\left(r_{1}(t)^{2}\right)} \text {. }
$$

This means that the terms under the summation sign in equation (14) can be expressed as acf, which leads to

$$
\begin{aligned}
E\left(r_{k+1}(t)^{2}\right)= & E\left(r_{k}(t)^{2}\right)+E\left(r_{1}(t)^{2}\right) \\
& +2 E\left(r_{1}(t)^{2}\right) \sum_{i=0}^{k-1} C(k-i) .
\end{aligned}
$$

Making use of the scaling law

$$
E\left(r_{k}(t)^{2}\right)=A k^{f(2)}
$$

yields the relation

$$
(k+1)^{f(2)}=k^{f(2)}+1+2 \sum_{i=0}^{k-1} C(k-i) .
$$

It can now be proved by complete induction that $C(k)$ has the form

$$
C(k)=\frac{(k+1)^{f(2)}}{2}-k^{f(2)}-\frac{(k-1)^{f(2)}}{2} .
$$

For $n=1$ this relation follows directly from equation (18). Now let's assume that equation (19) it is true up to a certain $k=n$. Then for $k=n+1$ solving equation (18) for $C(n+1)$ yields

$C(n+1)=\frac{(n+2)^{f(2)}-(n+1)^{f(2)}-1}{2}-\sum_{i=1}^{n} C(n+1-i)$.

By means of equation (18) the sum can be rewritten as

$$
\begin{aligned}
\sum_{i=1}^{n} C(n+1-i) & =C(n)+\sum_{i=1}^{n-1} C(n-i) \\
& =\frac{(n+1)^{f(2)}-(n)^{f(2)}-1}{2} .
\end{aligned}
$$

Inserting this into equation (19) proves the proposition.

The asymptotic behavior $(k>>1)$ can be found by expanding the right-hand-side of equation (19) up to second order around $k$. The first-order terms cancel, such that the final result is

$$
C(\tau)=2 H(2)(2 H(2)-1) \tau^{2 H-2},
$$

where $\tau=k \Delta$.

\section{References}

1. B.B. Mandelbrot, Journal of Business 36, 394 (1963)

2. U.A. Müller, M.M. Dacorogna, R.B. Olsen, O.V. Pictet, M. Schwarz, C. Morgenegg, Journal of Banking and Finance 14, 1189 (1990)

3. M.M. Dacorogna, R. Gençay, U.A. Müller, R.B. Olsen, O.V. Pictet, An Introduction to High-Frequency Finance (Academic Press, San Diego, CA, 2001) 
4. S. Ghashghaie, W. Breymann, J. Peinke, P. Talkner, Y. Dodge, Nature 381, 767 (1996)

5. J.F. Muzy, J. Delour, E. Bacry, Eur. Phys. J. B 17, 537 (2000)

6. W. Breymann, S. Ghashghaie, P. Talkner, International Journal of Theoretical and Applied Finance 3, 357 (2000)

7. B.B. Mandelbrot, Quantitative Finance 1, 113 (2001)

8. B.B. Mandelbrot, Quantitative Finance 1, 124 (2001)

9. B.B. Mandelbrot, Quantitative Finance 1, 427 (2001)

10. B.B. Mandelbrot, Quantitative Finance 1, 641 (2001)

11. R.H. Riedi, Long range dependence : theory and applications, edited by P. Doukhan, G. Oppenheim, M.S. Taqqu (Birkhäuser, 2002), pp. 625-715, ISBN: 0817641688

12. T.D. Matteo, T. Aste, M.M. Dacorogna, Physica A: Statistical Mechanics and its Applications 324, 183 (2003), proceedings of the International Econophysics Conference

13. T.D. Matteo, T. Aste, M.M. Dacorogna, Journal of Banking and Finance 29, 827 (2005)

14. T.D. Matteo, Quantitative Finance 7, 21 (2007)

15. E. Bacry, J. Delour, J.F. Muzy, Phys. Rev. E 64, 026103 (2001)

16. L. Calvet, A. Fisher, Journal of Econometrics 105, 27 (2001)

17. L. Calvet, A. Fisher, The Review of Economics and Statistics 84, 381 (2002)

18. R.N. Mantegna, H.E. Stanley, Nature 376, 46 (1995)

19. R.N. Mantegna, H.E. Stanley, Physica A 239, 255 (1997)

20. K. Kiyono, Z.R. Struzik, Y. Yamamoto, Phys. Rev. Lett. 96, 068701 (2006)

21. M. Bartolozzi, D. Leinweber, A. Thomas, Physica A: Statistical Mechanics and its Applications 350, 451 (2005)

22. J.L. McCauley, G.H. Gunaratne, K.E. Bassler, Physica A 379, 1 (2007)

23. W. Breymann, L. Kelly, E. Platen, Asia-Pacific Financial Markets 12, 1 (2006)

24. T. Le, E. Platen, The Journal of Risk Finance 7, 559 (2006)

25. W. Breymann, Internal document WAB.2000-07-31, Olsen \& Associates, Seefeldstrasse 233, 8008 Zürich, Switzerland (2000)

26. W. Breymann, A. Dias, P. Embrechts, Quantitative Finance 3, 1 (2003)
27. W. Breyman, hffinance, Available from URL https:// home.zhaw.ch/ ${ }^{\sim}$ bwlf / (2002)

28. J. Feder, Fractals, Physics of solids and liquids (Plenum Press, 1988)

29. B.B. Mandelbrot, Fractals and Scaling In Finance (Springer, 1997)

30. O.E. Barndorff-Nielsen, Scandinavian Journal of Statistics 5, $151(1978)$

31. O.E. Barndorff-Nielsen, P. Blaesild, in Statistical Distributions in Scientific Work, edited by C. Taillie, G. Patil, B. Baldessari (Reidel, Dordrecht, 1981), Vol. 4, pp. 19-44

32. E. Eberlein, U. Keller, Bernoulli 1, 281 (1995)

33. E. Eberlein, U. Keller, K. Prause, Journal of Business 71, 371 (1998)

34. B.M. Bibby, M. Sorensen, in Handbook of Heavy Tailed Distributions in Finance, edited by S.T. Rachev (Elsevier, Amsterdam, 2003), pp. 211-248

35. A.J. McNeil, R. Frey, P. Embrechts, Quantitative Risk Management: Concepts, Techniques, and Tools (Princeton University Press, Princeton, 2005)

36. N.H. Bingham, R. Kiesel, Quantitative Finance 2, 241 (2002)

37. K. Fergusson, E. Platen, Applied Mathematical Finance 13, 19 (2006)

38. S. Kotz, T.J. Kozubowski, K. Podgórski, The Laplace Distribution and Generalizations (Birkhäuser, 2001)

39. B.M. Bibby, M. Sørensen, Finance \& Stochastics 2, 25 (1997)

40. O.E. Barndorff-Nielsen, Scandinavian Journal of Statistics 24, 1 (1997)

41. S. Raible, Ph.D. thesis, Universität Freiburg i. Br., 2000, http://deposit.ddb.de/cgi-bin/dokserv? idn $=961285192$

42. O.E. Barndorff-Nielsen, Finance and Stochastics 2, 41 (1997)

43. O.E. Barndorff-Nielsen, N. Shephard, Continuous Time Approach to Financial Volatility (Cambridge University Press, Cambridge, 2008)

44. E. Platen, D. Heath, A Benchmark Approach to Quantitative Finance (Springer, Berlin, 2006) 